\title{
Biological Pacemaker
}

\author{
Uzzal Kanti Das ${ }^{1}$, Syed Ali Ahsan², Mohammad Saifullah Patwary², KMHS Sirajul Haque², \\ S. M. Mustafa Zaman², Md. Harisul Hoque ${ }^{2}$ \\ ${ }^{1}$ Medicine General Hospital, Noakhali. \\ 2 Department of Cardiology, Bangabandhu Sheikh Mujib Medical University, Dhaka \\ Address for correspondence \\ Dr. Uzzwal Kanti Das, Senior Consultant, Medicine General Hospital, Noakhali. \\ e-mail : draliahsan@yahoo.com
}

\begin{abstract}
Electronic cardiac pacemakers are the definitive treatment of sinoatrial node (SAN) dysfunction and high degree atrioventricular block. But they have limitations like limited battery life, need for lead implantation into heart and lack of response to autonomic and physiologic demands on the heart. Genetically engineered biological pacemakers could be a possible alternative to electronic pacemakers. The strategies include upregulation of $\beta-1$ adrenergic receptors, conversion of cardiomyocyte into pacemaker cells and stem cell therapy. Investigators created biological pacemakers in animal models by gene therapy targeting $\mathrm{I}_{\mathrm{f}}$ current $(\mathrm{HCN})$ and inward rectifier current $\left(\mathrm{I}_{\mathrm{kI}}\right)\left(\mathrm{K}_{\mathrm{ir}}\right.$ 2.1). They delivered pacemaker current gene into animal heart by using viral vectors and human mesenchymal stem cells (hMSC). The limitations noted are longevity and stability of pacemaker genes and the chance of migration and tumour formation after stem cells therapy.
\end{abstract}

Key words : Sinoatrial node (SAN), HCN-2 gene, If current, inward rectifier current (Ikl), human embryonic stem cells.

\section{Introduction}

Despite the fact that electronic pacemaker is the definitive treatment of bradyarrhythemias like sick sinus syndrome, sinoatrial block atrioventricular block and congenitical heart block, time has come to think of a biological pacemaker. ${ }^{1}$ Because electronic pacemakers are palliative, not suitable in a growing child as a long term treatment, has a fixed life necessitating monitoring and replacement, prone to infection and thromboembolism, chances of electromechanical nonfunctioning, may be affected by strong electro magnetic field like MRI and non responsive to emotion and exercise. ${ }^{1,2}$ Genetically engineered pacemakers would be a possible alternative to electronic pacemaker as a curative one and emotions responsive to autonomic uervous system, exercise and emotion. With the advancement of cardiac gene and cell therapy investigators have prompted to create a biological pacemaker by gene and cell transfer techniques which would offer the possibility to design and create both a pacemaker to initiate the heart beat, and an excitable tissue capable of propagating, impulse from atrium to ventricle in complete heart block. The strategies include upregulation of beta adrenergic receptors by injection of plasmids encoding the $\beta-2$ adrenergic receptors into the atria of animals, conversion of myocytes into pacemaker cells via insertion of viral vectors encoding pacemaker gene into the myocardial cells of animals in heart block and use of stem cells as platforms to deliver pacemaker genes native to or implanted in the stem cells to the recipient heart. ${ }^{3}$

\section{Discussion}

Though SAN is a relatively small structure it has a very complex morphological and electrophysiological properties. The normal pacemaker physiology of SAN is contributed by population of cells with a gradient of morphological and electrophysiological properties in SAN. Its anatomical structure contains a connective tissue barrier and transitional cells. The basis of sinus node pacemaker function is the target of all strategies building biological pacemakers. During phase 4 , of action potential cells in the sinoatrial node (SAN), atrioventricular node (AVN) and the Purkinje system undergo slow diastolic depolarization. ${ }^{4}$ Various ion currents interplay in a complex manner to generate this depolarization. Of these $I_{f}$ (funny) current plays the dominant role in the initiation, control and the rate of diastolic depolarization. ${ }^{4}$ The pacemaker potential $\left(\mathrm{I}_{\mathrm{f}}\right)$ is generated via hyperpolarisation induced opening of an ion channel of the NCN (Hyperpolarisation- activated, cyclic nucleotide gated) family which has four isoforms, ${ }^{1,5} \mathrm{HCN} 1-4 \mathrm{HCN} 1, \mathrm{HCN} 2$ and $\mathrm{HCN} 4$ are present in the heart and are activated upon hyperpolarisation and directly bind to Cyclic AMP (cAMP) through a cAMP binding site and is sensitive to modulation by catecholamines. The cAMP binding shifts activation towards more positive voltages, effectively increasing inward sodium current at a given voltage. HCN2 has faster kinetics than HCN4 and binds strongly to cAMP than HCN1. ${ }^{5}$ Thus initial studies focused on HCN2 gene to build a biological pacemaker. Other ion channels also contribute to pacemaker potential like $\mathrm{Na}+$ calcium exchanger, both $\mathrm{T}$ and L-type calcium currents. 1,5 
Repolarisation, occurs because of outward potassium current. Any intervention that increases inward current or decreases outward current will increase pacemaker rate. ${ }^{1}$ Stimulation of beta adrenergic receptors results in faster rate via catecholamine binding to $\beta$ - adrenergic receptors ( $\beta$-AR) this causes cAMP bindings to HCN channel resulting in rapid membrane depolarization and increase pacemaker rate. ${ }^{1,6}$

In recent years a number of studies carried out to build a biological pacemaker by (i) upregulating adrenergic receptors, (ii) down regulation of inward rectifier potassium current (Ik1) and (iii) over expression of HCN2 channel $\left(\mathrm{I}_{\mathrm{f}}\right.$ current) in animaIs. ${ }^{7}$ Edelberg et al. ${ }^{6}$ first explored the $\beta-2$ AR as a target for gene transfer and demonstrated that injection of the $\beta-2$ AR construct (plasmids) in the right atrium of mouse hearts increased endogenous heart rate by $40 \%$ by 2 days. Subsequently injection of the $\beta-2$ AR construct in the right atrium of pigs resulted in a heart rate increase of $50 \%$ and expression of encoded $\beta-2$ AR gene. Other investigators developed a biological pacemaker by adenovral gene transfer to enhance HCN2. Injection of adenovirus encoding HCN2-GFP (Green Fluorescent protein) in the left atrium (LA) of dogs resulted in spontaneous LA rhythm in 4 of 4 following sinus arrest induced by vagal stimulation. The left atrial myocytes transfected with $\mathrm{HCN} 2+$ GFP genes exhibited $>500$ times larger $\mathrm{I}_{\mathrm{f}}$ current than non-expressing atrial myocytes. ${ }^{8}$ Mike et al. ${ }^{9}$ developed a biological pacemaker by suppressing $\mathrm{I}_{\mathrm{ki}}$ (Inward rectifier potassium current encoded by $\mathrm{k}_{\mathrm{ir}}{ }^{2}$ gene family) in ventricular myocytes. They created a dominant negative construct that suppress the $\mathrm{I}_{\mathrm{ki}}$ current when transfected and co-expressed with $\mathrm{k}_{\mathrm{ir}^{-2}}$.1 AAA resulted in a dominant negative constructing pores. $\mathrm{K}_{\mathrm{ir}}-2.1 \mathrm{AAA}$ was then packaged with GFP in adenovirus that would allow expression of both dominant negative kir-2.1 constructs and GFP as a marker. Adenovirus was then injected in the LV cavity of guinea pigs. Myocytes isolated 3-4 days later exhibited decreased $\mathrm{I}_{\mathrm{ki}}$ by $50-90 \%$. Those cells with $>80 \%$ reduction of $l_{\mathrm{ki}}$ showed pacemaker phenotype. Other investigators prepared engineered tissue constructs from, skeletal myoblasts when implanted to build atrioventricular bridges carry impulses from atrium to ventricle in experimentally induced complete heart block and normal sinus node funetion. 10

Alternatively stem cells are used as platforms to deliver pacemaker genes native to or implanted in stem cells to the recipient' heart. Human mesenchymal stem cells (hMSC) transfected with HCN2 genes by electroporation (thus avoiding viral vectors) and then injection of those cells into the ventricle resulted in formation of gap junction with adjacent myocytes and delivery of pacemaker current to them Kanai $\mathrm{S}$ et al. ${ }^{8}$ emphasizes the role of the decreased level of expression of $\mathrm{I}_{\mathrm{ki}}$ to ensure that the coupled myocyte- stem cells (transfected with HCN2 genes) become self-oscillatory and this will be helpful to create a new biological pacemaker.

Another approach involves the administration of human embryonic stem cells forced into a cardiogenic lineage (pacemaker cells) when injected into myocardium of pigs in heart block generate a pacemaker current and produce stable idioventricular rhythm. ${ }^{1,4}$ Fetal and neonatal cell transplants experiments are also ongoing. ${ }^{11}$

While efforts are ongoing to build a biological pacemaker, issues of safety of viruses and cell platform must be considered as there is a possibility, of tumour formation and potential for migration of transplanted cells to other sites of the body.

\section{Conclusion}

Much has been learnt in preclinical studies and yet to be learnt are safety of viral vectors and stem cell therapy in future preclinical studies. We can hope in near future a safe and effective biological pacemaker will be available for use in human being.

\section{References}

1. Michael R Rosen, Biological pacemaker: a concept whose time has come... or is coming. Heart. 2007;93: 145-46

2. Rosen MR. Brink, PR, Cohen IS et al. Genes, stem cell and biological pacemakers. Cardiovasc Res 2004;64: 12-23

3. Analisa Bucchi. Alexi N. Plotuikov, Iryna Shalapakova et al. Wild-Type and Mutant HCN Channels in a Tandem Biologieai Electronic Cardiac pacemaker. Circulation 2006;114:992-99

4. Traian M. Anghel. Steven M. Pogwirzd. Creating a cardiac pacemaker by gene therapy. Med Bio Eng comput. 2007;45: 45-55

5. Biel M, Schneider A, Wahl C. Cardiac IICN channels: structure, function and modulation. Trends Cardiovasc Med 2002;12:2002-16

6. J.M. Edelberg, D.T. Huang, M.E. Josephson et at. Molecular Enhancement of Porcine cardiac chronotrophy. Heart 2001; 86:559-62

7. G. Rajesh and Johnson Francis. Biological Pacemaker. Indian packing and Eelctrophygiol J. 2006;6 (1): 1-5

8. S Kanani et at. Genetically engineered condiac pacemaker: stem cell transfected with HCN 2 gene and myoeytes. A model physics letters 2007

9. Miake J. Marban E. Nuss HB. Functional role of inward rectifier current in heart probed by Kir 2. 1 overexpression and dominant negative suppression. J Clin Invest 2003;1 11:1529-36

10. Choi YH, Starnm C, Hammer PE et al. Cardiac conduction through engineered tissue. Am J Pathol 2006;169:72-85.

11. Ruhparwar A, Tebbenjohanns J, Niehaus $\mathrm{M}$ et at. Transplanted fetal cardiomyocytes as cardiac pacemaker. Eur J Cardiothorac Surg 2002;21:853-57 